\title{
CHEBYSHEV POLYNOMIALS ON JULIA SETS AND EQUIPOTENTIAL CURVES FOR THE FAMILY $P(z)=z^{d}-c$
}

\author{
YINGQING XIAO ${ }^{凶}$ and WEIYUAN QIU
}

(Received 30 January 2006; accepted 23 April 2007)

Communicated by P. C. Fenton

\begin{abstract}
It is shown that the $d^{n}$ th Chebyshev polynomials on the Julia set $J_{P}$, and on the equipotential curve $\Gamma_{P}(R)$, of the polynomial $P(z)=z^{d}-c$, are identical and exactly equal to the $n$th iteration of $P(z)$ itself. As an application, the capacity of the Julia set $J_{P}$ is deduced, a result that was first obtained by Brolin.
\end{abstract}

2000 Mathematics subject classification: primary 37F99; secondary 32H02.

Keywords and phrases: Chebyshev polynomials, Julia set, equipotential curve.

\section{Introduction}

For a compact subset $K$ of the complex plane $\mathbb{C}$ with at least $n$ elements, there exists a unique complex polynomial $F_{n}$ of degree $n$ and with leading coefficient 1 such that

$$
\max _{z \in K}\left|F_{n}(z)\right|=\inf \left\{\max _{z \in K}\left|z^{n}+a_{1} z^{n-1}+\cdots+a_{n}\right|: a_{1}, \ldots, a_{n} \in \mathbb{C}\right\} .
$$

The polynomial $F_{n}$ is called the $n$th Chebyshev polynomial on the set $K$. For example, if $K$ is the unit circle $\{z \in \mathbb{C}:|z|=1\}$, then $F_{n}(z)=z^{n}$. If $K$ is the segment $[0,4]$ on the real axis, then $F_{n}$ is the classic $n$th Chebyshev polynomial, that is $F_{n}(z-2)=w^{n}+\left(1 / w^{n}\right)$, where $z=w+(1 / w)+2,|w|>1$. In [2], Barnsley et al. showed that the $2^{n}$ th Chebyshev polynomial on the Julia set of $T(z)=(z-\lambda)^{2}$ is $T^{n}(z)-\lambda$, where $T^{n}(z)=T\left(T^{n-1}(z)\right)$ for $n \in\{1,2,3, \ldots\}$ and $T^{0}(z)=z$. In this case, the Julia set can be disconnected. Another example of Chebyshev polynomials on disconnected compact sets was given by Fischer [7]. In this paper, we generalize the conclusion of [2] to the Julia sets of polynomials $P(z)=z^{d}-c$ for $d \geq 2$ and $c \in \mathbb{C}$.

This work is supported by NNSF No. 10571028.

(c) 2009 Australian Mathematical Society 1446-7887/2009 \$16.00 
Theorem 1. Let $J_{P}$ denote the Julia set of $P(z)=z^{d}-c$ for $d \geq 2$ and $c \in \mathbb{C}$. Then the $d^{n}$ th Chebyshev polynomial on $J_{P}$ is $P^{n}(z)$, where $P^{n}(z)=P\left(P^{n-1}(z)\right)$ for $n \in\{1,2,3, \ldots\}$ and $P^{0}(z)=z$.

Another problem concerns the Chebyshev polynomials on the equipotential curves for a compact set in $\mathbb{C}$. Let $K$ be a compact subset of $\mathbb{C}$ and $A_{K}$ be the unbounded component of $\mathbb{C} \backslash K$. By definition, an equipotential curve $\Gamma_{K}(R)$ of level $R>1$ for $K$ is the set

$$
\Gamma_{K}(R)=\left\{z \in A_{K}: G_{K}(z)=\log R\right\}, \quad R>1,
$$

where $G_{K}(z)$ is Green's function for $A_{K}$ with pole at $\infty$.

For the unit circle $\{z:|z|=1\}$, the equipotential curves are given by $\{z:|z|=R\}$ for $R>1$. It is obvious that $F_{n}(z)=z^{n}$ is also the $n$th Chebyshev polynomial on any circle $\{z:|z|=R\}$. This shows that the Chebyshev polynomials on $\{z:|z|=1\}$ and on $\{z:|z|=R\}(R>1)$ are identical. For the real segment [0,4], the equipotential curves are ellipses with foci 0 and 4. It was proved by Faber [5] that the classical Chebyshev polynomials are also polynomials with minimal uniform norm on such curves, and are thus the Chebyshev polynomials on the equipotential curves. It is reasonable to ask if it is always true for a compact planar set $K$ that the Chebyshev polynomials on $K$ (or at least a subsequence of them) are also the Chebyshev polynomials on equipotential curves $\Gamma_{K}(R)$ for any $R>1$ (refer to [11]). Fischer [7] showed that this is the case when $K$ is the union of a certain pair of disjoint real segments. In [11], Stawiska studied the problem for Julia sets of quadratic polynomials $T_{\lambda}(z)=(z-\lambda)^{2}$ and proved that, when $\lambda \in[0,2]$, the $2^{n}$ th Chebyshev polynomial $T^{n}(z)-\lambda$ on the Julia set of $T_{\lambda}$ is also the $2^{n}$ th Chebyshev polynomial on the equipotential curves. Note that the Julia sets of $T_{\lambda}(z)$ are connected in this case, and the unit circle and the real line segment $[0,4]$ appear as special cases. In this paper, we show that the restriction $\lambda \in[0,2]$ is not required. In fact, we extend Stawiska's result [11] to the Julia sets of polynomials $P(z)=z^{d}-c$ with degree $d \geq 2$ and any $c \in \mathbb{C}$.

TheOREM 2. Let $P(z)=z^{d}-c$ with $d \geq 2$ and $c \in \mathbb{C}$. Let $J_{P}$ be the Julia set of $P(z)$. Then the $d^{n}$ th Chebyshev polynomial on the equipotential curve $\Gamma_{P}(R)$ for $R>1$ is $P^{n}(z)$, where $P^{n}(z)=P\left(P^{n-1}(z)\right)$ for $n \in\{1,2,3, \ldots\}$ and $P^{0}(z)=z$.

From Theorems 1 and 2, we have the following conclusion.

Corollary 3. For the polynomial $P(z)=z^{d}-c$ with $d \geq 2$ and $c \in \mathbb{C}$, the $d^{n}$ th Chebyshev polynomial on the Julia set $J_{P}$ is also the Chebyshev polynomial on any equipotential curve of level $R>1$ for $J_{P}$.

Note that in our result the Julia set $J_{P}$ can be disconnected.

The paper is organized as follows. In Section 2 we introduce some definitions and results from holomorphic dynamics, which will be used in the remainder of the paper. For general background in complex dynamics, we refer to [3, 4, 9]. The proofs of Theorem 1 and 2 appear in Section 3. In Section 4, as an application of the results given in Section 3, we calculate the capacities of the Julia sets and the equipotential curves 
for the family $P(z)=z^{d}-c$. The capacities of the Julia sets of monic polynomials were first given by Brolin [4].

\section{Preliminaries}

Let $P: \mathbb{C} \rightarrow \mathbb{C}$ be a polynomial of degree $d \geq 2$. Let $P^{n}(z)$ be the $n$th iteration of the polynomial $P(z)$, namely,

$$
P^{n}(z)=P\left(P^{n-1}(z)\right) \quad \text { for } n \in\{1,2,3, \ldots\} \text { and } P^{0}(z)=z .
$$

The Julia set $J_{P}$ for $P$ is defined to be the set of points at which the family of iterates of $P$ fails to be a normal family in the sense of Montel [10]. Equivalently, the Julia set is the closure of the union of repulsive fixed points of $P^{n}(z)$ for all $n \in\{1,2,3, \ldots\}$ (see $[4,6,8]$ ). For properties of Julia sets, we refer to $[3,4,9]$.

It is well known that the Julia set is a compact subset of $\mathbb{C}$ and there exists no interior point in $J_{P}$. The set $J_{P}$ is completely invariant under $P$, in other words,

$$
P^{-1}\left(J_{P}\right)=P\left(J_{P}\right)=J_{P} .
$$

It follows that, for any positive integer $n$, the maximum modulus of $P^{n}(z)$ on $J_{P}$ is given by

$$
\max _{z \in J_{P}}\left|P^{n}(z)\right|=\max _{z \in J_{P}}|z| .
$$

Let $A_{P}(\infty)$ denote the set of points $z \in \mathbb{C}$ such that the iterate sequence $\left\{P^{n}(z)\right\}$ is unbounded. Equivalently,

$$
A_{P}(\infty)=\left\{z \in \mathbb{C}: P^{n}(z) \rightarrow \infty\right\} .
$$

The set $A_{P}(\infty)$ is called the attractive basin of $\infty$, is an open subset of $\mathbb{C}$ and contains a neighborhood of $\infty$.

The complement $K_{P}$ of $A_{P}(\infty)$, that is,

$$
K_{P}=\left\{z \in \mathbb{C}: P^{n}(z) \nrightarrow \infty\right\},
$$

is called the filled Julia set, and is compact in $\mathbb{C}$. Both $A_{P}(\infty)$ and $K_{P}$ are completely invariant under $P$. Their common boundary

$$
\partial K_{P}=\partial A_{P}(\infty)=J_{P}
$$

is the Julia set of the polynomial $P$. It is well known from Fatou [6] that if all critical points of $P$, that is, the points satisfying $P^{\prime}(z)=0$, belong to $K_{P}$, then $K_{P}$ and $J_{P}$ are connected. Otherwise, $J_{P}$ is disconnected.

Define the function

$$
G: \mathbb{C} \rightarrow \mathbb{R}^{+} \cup\{0\}
$$

by

$$
G(z)=\lim _{n \rightarrow \infty} \frac{1}{d^{n}} \log _{+}\left|P^{n}(z)\right|,
$$

where $\log _{+}|z|=\max \{0, \log |z|\}$. It is known (see [9]) that $G(z)$ is harmonic in 
$\mathbb{C} \backslash K_{P}$ and $K_{P}$ is the set of zeros of $G(z)$. In fact, $G(z)$ is Green's function for $\mathbb{C} \backslash K_{P}$ with pole at $\infty$. Consequently, for $R>1$, the curve

$$
\Gamma_{P}(R)=\left\{z \in \mathbb{C} \backslash K_{P}: G(z)=\log R\right\}
$$

is the equipotential curve of level $R$. From the definition,

$$
P\left(\Gamma_{P}(R)\right)=\Gamma_{P}\left(R^{d}\right) \quad \text { and } \quad \Gamma_{P}(R)=P^{-1}\left(\Gamma_{P}\left(R^{d}\right)\right) .
$$

It follows that, for any positive integer $n$,

$$
\max _{z \in \Gamma_{P}(R)}\left|P^{n}(z)\right|=\max _{z \in \Gamma_{P}\left(R^{d^{n}}\right)}|z|, \quad R>1 .
$$

When the Julia set $J_{P}$ is connected, then there exists a conformal mapping $\Phi: \mathbb{C} \backslash K_{P} \rightarrow\{w \in \mathbb{C}:|w|>1\}$ satisfying $\lim _{z \rightarrow \infty}(\Phi(z) / z)=1$. In this case, $G(z)=\log |\Phi(z)|$ for $z \in \mathbb{C} \backslash K_{P}$. So the equipotential curves are given by

$$
\Gamma_{P}(R)=\{z:|\Phi(z)|=R\},
$$

which are simple closed analytic curves surrounding $J_{P}$ for $R>1$.

\section{Proof of theorems}

In this section, we restrict our attention to the family of polynomials $P(z)=z^{d}-c$, where $d \geq 2$ is an integer and $c \in \mathbb{C}$. Let $\omega_{k}=e^{(2 \pi k / d) i}$ for $k=0,1, \ldots, d-1$ be the roots of the equation $z^{d}=1$. Then $P\left(\omega_{k} z\right)=P(z)$ for any $k=0,1, \ldots, d-1$. It follows that both the Julia set $J_{P}$ and the equipotential curves $\Gamma_{P}(R)$ of $P(z)$ are invariant under multiplication by any $\omega_{k}$. That is, we have the following lemma.

LEMMA 4. $z \in J_{P}\left(z \in \Gamma_{P}(R)\right)$ implies that, for every $k, \omega_{k} z \in J_{P}\left(\omega_{k} z \in \Gamma_{P}(R)\right.$, respectively).

To obtain the Chebyshev polynomials on $J_{P}$ and $\Gamma_{P}(R)$, we need the following lemma.

LEMMA 5. Let $\omega_{0}, \omega_{1}, \ldots, \omega_{d-1}$ be the roots of the equation $z^{d}=1$. Then for any integer $n>0$, and $a, a_{1}, a_{2}, \ldots, a_{n} \in \mathbb{C}$ and $l_{1}, l_{2}, \ldots, l_{n} \in \mathbb{N}$ with $1 \leq l_{j} \leq d-1$, for $j=1,2, \ldots, n$, there exists at least one integer $k$ with $0 \leq k \leq d-1$ such that

$$
\left|a+a_{1} \omega_{k}^{l_{1}}+a_{2} \omega_{k}^{l_{2}}+\cdots+a_{n} \omega_{k}^{l_{n}}\right| \geq|a| .
$$

PROOF. Let $S_{k}=a+a_{1} \omega_{k}^{l_{1}}+a_{2} \omega_{k}^{l_{2}}+\cdots+a_{n} \omega_{k}^{l_{n}}$. Then

$$
\left|\sum_{k=0}^{d-1} S_{k}\right|=\left|\sum_{k=0}^{d-1}\left(a+a_{1} \omega_{k}^{l_{1}}+a_{2} \omega_{k}^{l_{2}}+\cdots+a_{n} \omega_{k}^{l_{n}}\right)\right|=\left|d a+\sum_{j=1}^{n} a_{j} \sum_{k=0}^{d-1} \omega_{k}^{l_{j}}\right| .
$$

Note that $\sum_{k=0}^{d-1} \omega_{k}^{l_{j}}=0$ for $1 \leq l_{j} \leq d-1$. Thus there exists a $k$, with $0 \leq k \leq d-1$, such that $\left|S_{k}\right| \geq|a|$.

Now we give the proof of Theorem 1. 
Proof OF THEOREM 1. Let $Q$ be a polynomial of degree $d^{n}$ with leading coefficient 1 . Denote by $W$ the set of $d$ roots of the equation $z^{d}=1$, that is,

$$
W=\left\{\omega_{k}=e^{(2 \pi k / d) i}: k=0,1, \ldots, d-1\right\} .
$$

Let $z_{0} \in J_{P}$ satisfy $\max _{z \in J_{P}}\left|P^{n}(z)\right|=\max _{z \in J_{P}}|z|=\left|z_{0}\right|$. The purpose is to show that there exists a root $z$ of $P^{n}(z)=w_{0} z_{0}$ for some $w_{0} \in W$, such that $|Q(z)| \geq\left|z_{0}\right|$.

For a given integer $j$, with $0<j \leq d^{n}-1$, we can choose $\left(l_{1}, n_{1}\right), \ldots,\left(l_{j}, n_{j}\right) \in$ $\mathbb{N} \times \mathbb{N}$ satisfying $0 \leq n_{1}<n_{2}<\cdots<n_{j}<n$ and $1 \leq l_{1}, l_{2}, \ldots, l_{j} \leq d-1$ such that

$$
j=l_{1} d^{n_{1}}+l_{2} d^{n_{2}}+\cdots+l_{j} d^{n_{j}} .
$$

Set $U_{0}(z) \equiv 1$. Thus, we can represent $Q$ as the sum

$$
Q(z)=P^{n}(z)+\sum_{j=0}^{d^{n}-1} a_{j} U_{j}(z)
$$

where $a_{j}$ are complex coefficients and

$$
U_{j}(z)=\left(P^{n_{1}}(z)\right)^{l_{1}}\left(P^{n_{2}}(z)\right)^{l_{2}} \ldots\left(P^{n_{j}}(z)\right)^{l_{j}} \quad \text { for } j>0 .
$$

When $j>0$, we define the index of the polynomial $U_{j}$ to be the integer $n_{1}$, denoted by index $U_{j}=n_{1}$. The index of $U_{0} \equiv 1$ is set to be $n$. Then we regroup terms by index, that is,

$$
Q(z)=P^{n}(z)+\sum_{k=0}^{n} q_{k}(z)
$$

where $q_{k}(z)=\sum_{\text {index } U_{j}=k} a_{j} U_{j}(z)$.

Lastly, we introduce

$$
L_{m}(z)=P^{n}(z)+\sum_{k=n-m}^{n} q_{k}(z) \text { for } m=0,1,2, \ldots, n \text {. }
$$

We have $L_{m}(z)=L_{m-1}(z)+q_{n-m}(z)$ and $L_{n}(z)=Q(z)$.

Note that any root $z$ of the equation $P^{n}(z)=w_{0} z_{0}$ can be written as

$$
z=w_{n} \sqrt[d]{c+w_{n-1} \sqrt[d]{c+\cdots+w_{1} \sqrt[d]{c+w_{0} z_{0}}}}
$$

where $w_{j} \in W$ for $j=0,1, \ldots, n$. We claim that, for each $k=0,1,2, \ldots, n$, there are unit roots $w_{j} \in W$ for $j=0,1,2, \ldots, k$, such that for any $w_{j} \in W$ with $j=k+1, \ldots, n$, we have $\left|L_{k}(z)\right| \geq\left|z_{0}\right|$ where

$$
z=w_{n} \sqrt[d]{c+w_{n-1} \sqrt[d]{c+\cdots+w_{1} \sqrt[d]{c+w_{0} z_{0}}}}
$$


In particular, for $k=n$, there will be a point $z$ on $J_{P}$ such that $|Q(z)|=\left|L_{n}(z)\right| \geq\left|z_{0}\right|$, which is what we need to show.

We will prove the claim by induction. First let $k=0$. No matter how $w_{0}, w_{1}, \ldots, w_{n}$ are chosen to determine $z$,

$$
\left|L_{0}(z)\right|=\left|P^{n}(z)+a_{0}\right|=\left|w_{0} z_{0}+a_{0}\right| .
$$

Now we can choose a $w_{0} \in W$ to ensure $\left|w_{0} z_{0}+a_{0}\right| \geq\left|z_{0}\right|$.

Let us assume that the claim is true for $k=m-1<n$. We will show that it is also true for $k=m$. Suppose that $w_{0}, w_{1}, \ldots, w_{m-1} \in W$ are taken as in the statement for $k=m-1$, and we defer choosing $w_{m}$. With $z$ as in the claim,

$$
\begin{aligned}
P^{n-m}(z) & =w_{m} \sqrt[d]{c+w_{m-1} \sqrt[d]{c+\cdots+w_{1} \sqrt[d]{c+w_{0} z_{0}}}} \\
& =w_{m} \times\left(\text { quantity independent of } w_{m}\right) .
\end{aligned}
$$

For $n-m<k \leq n$,

$$
P^{k}(z)=w_{n-k} \sqrt[d]{c+w_{n-k-1} \sqrt[d]{c+\cdots+w_{1} \sqrt[d]{c+w_{0} z_{0}}}},
$$

which is also independent of $w_{m}$. So

$$
\begin{aligned}
q_{n-m}(z) & =\sum_{\text {index }} a_{j}=n-m \\
& =\sum_{i}\left(P_{j}^{n-m}(z)\right)^{l_{i}} \times\left(\text { terms independent of } w_{m}\right) \\
& =\sum_{i} w_{m}^{l_{i}} \times\left(\text { terms independent of } w_{m}\right) .
\end{aligned}
$$

Now $w_{0}, w_{1}, \ldots, w_{m-1}$ have been determined and $L_{m-1}(z)$ is independent of $w_{m}$. By Lemma 5, we can choose $w_{m} \in W$ such that

$$
\left|L_{m}(z)\right|=\left|q_{n-m}(z)+L_{m-1}(z)\right| \geq\left|L_{m-1}(z)\right| \geq\left|z_{0}\right| .
$$

The second inequality follows from the inductive hypothesis. The claim is proved. For $m=n$, we have $|Q(z)|=\left|L_{n}(z)\right| \geq\left|z_{0}\right|=\max _{z \in J_{P}}\left|P^{n}(z)\right|$. This completes the proof.

PROOF OF ThEOREM 2. The proof is quite similar to the proof of Theorem 1. To show that $P^{n}(z)$ is the $d^{n}$ th Chebyshev polynomial on the equipotential curve $\Gamma_{P}(R)$ for $R>1$, we take $z_{0}$ such that $\left|z_{0}\right|=\max _{z \in \Gamma_{P}\left(R^{d^{n}}\right)}|z|$. Since $\Gamma_{P}(R)=P^{-n}\left(\Gamma_{P}\left(R^{d^{n}}\right)\right)$, we have $\max _{z \in \Gamma_{P}(R)}\left|P^{n}(z)\right|=\left|z_{0}\right|$. Noting that the equipotential curves are invariant under multiplication by $\omega_{k}$, all roots of the equation $P^{n}(z)=w_{0} z_{0}$ for some $w_{0} \in W$ are in $\Gamma_{P}(R)$. So, as in the proof of Theorem 1, for any polynomial $Q(z)$ of degree $d^{n}$ with leading coefficient one, we need only to show that there exists a root $z$ of $P^{n}(z)=w_{0} z_{0}$ for some $w_{0} \in W,|Q(z)| \geq\left|z_{0}\right|$. This follows the proof of Theorem 1 . We omit the details. 
Corollary 3 is a direct consequence of Theorems 1 and 2 .

\section{Application}

Brolin [4] proved that the capacity of the Julia set for a complex polynomial $P$ of degree $d$ with leading coefficient one is 1 . For the family $P(z)=z^{d}-c$, Theorem 1 leads to a new proof of this result. To see this, we recall some definitions and results on capacity, which can be found in [1].

Let $E$ be a compact set in the complex plane $\mathbb{C}$. The diameter of order $n$ for $E$ is defined by

$$
d_{n}(E)=\max \left\{\prod_{i<j}\left|z_{i}-z_{j}\right|^{(2 / n(n-1))}: z_{i} \in E, i=1, \ldots, n\right\} .
$$

It is readily seen that $d_{n-1}(E) \leq d_{n}(E)$, so the limit $\lim _{n \rightarrow \infty} d_{n}(E)$ exists. We define $d_{\infty}(E)=\lim _{n \rightarrow \infty} d_{n}(E)$ to be the transfinite diameter of $E$.

If $P_{n}(z)$ is the $n$th Chebyshev polynomial on $E$, we denote its maximum modulus on $E$ by $\rho_{n}^{n}(E)$. Then we have the following proposition (see [1]).

Proposition 6. $\lim _{n \rightarrow \infty} \rho_{n}(E)=d_{\infty}(E)$.

Let $\mu$ be a positive mass distribution on $E$ of finite total mass. The logarithmic potential on $E$ is defined by

$$
u(z)=\int_{E} \log \frac{1}{|z-\zeta|} d \mu(\zeta)
$$

and the energy integral is

$$
I(\mu)=\iint_{E \times E} \log \frac{1}{|z-\zeta|} d \mu(\zeta) d \mu(z) .
$$

Set

$$
V=\inf _{\mu, \mu(E)=1} I(\mu) .
$$

Then the capacity $\gamma(E)$ of $E$ is defined by

$$
\gamma(E)=e^{-V}
$$

The following proposition tells us the relation between the capacity and the transfinite diameter (see [1]).

PROPOSITION 7. The capacity of a closed bounded set is equal to its transfinite diameter.

From Propositions 6 and 7, we have the following theorem.

THEOREM 8. For the family $P(z)=z^{d}-c, \gamma\left(J_{P}\right)=1$ and $\gamma\left(\Gamma_{P}(R)\right)=R$. 
Proof. From Theorem 1, we know that the $d^{n}$ th Chebyshev polynomial on $J_{P}$ is $P^{n}(z)$ and

$$
\rho_{d^{n}}^{d^{n}}\left(J_{P}\right)=\max _{z \in J_{p}}\left|P^{n}(z)\right|=\max _{z \in J_{p}}|z|=\left|z_{0}\right| .
$$

It follows from Propositions 6 and 7 that

$$
\gamma\left(J_{P}\right)=d_{\infty}\left(J_{P}\right)=\lim _{n \rightarrow \infty} \rho_{d^{n}}(E)=\lim _{n \rightarrow \infty} \sqrt[d^{n}]{\left|z_{0}\right|}=1 .
$$

From Theorem 2, the $d^{n}$ th Chebyshev polynomial on $\Gamma_{P}(R)$ is $P^{n}(z)$. Let $z_{n}$ be in $\Gamma_{P}\left(R^{d^{n}}\right)$ and it satisfies $\max _{z \in \Gamma_{P}\left(R^{d^{n}}\right)}|z|=\left|z_{n}\right|$. Then, as above,

$$
\rho_{d^{n}}^{d^{n}}\left(\Gamma_{P}(R)\right)=\max _{z \in \Gamma_{P}(R)}\left|P^{n}(z)\right|=\left|z_{n}\right|
$$

By definition, $\log G\left(z_{n}\right)=\log R^{d^{n}}=d^{n} \log R$. It is evident that $z_{n} \rightarrow \infty$ as $n \rightarrow \infty$ and $\log G(z) \sim \log |z|$ as $z \rightarrow \infty$. We have

$$
\log \left|z_{n}\right| \sim d^{n} \log R \quad \text { as } n \rightarrow \infty
$$

This implies that

$$
\lim _{n \rightarrow \infty} \frac{1}{d^{n}} \log \left|z_{n}\right|=\log R .
$$

So we have, from Propositions 6 and 7 ,

$$
\gamma\left(\Gamma_{P}(R)\right)=\lim _{n \rightarrow \infty} \rho_{d^{n}}\left(\Gamma_{P}(R)\right)=\lim _{n \rightarrow \infty} \sqrt[d^{n}]{\left|z_{n}\right|}=R
$$

This completes the proof.

\section{References}

[1] L. V. Ahlfors, Conformal Invariants, Topics in Geometric Function Theory (McGraw-Hill Book Company, 1973).

[2] M. F. Barnsley, J. S. Geronimo and A. N. Harrington, 'Some tree-like Julia sets and Padé approximations', Lett. Math. Phys. 7 (1983), 186-279.

[3] A. F. Beardon, Iteration of Rational Functions (Springer, Berlin, 1991).

[4] H. Brolin, 'Invariant sets under iteration of rational functions', Ark. Mat. 6 (1965), 103-144.

[5] G. Faber, 'Über Tschebyscheffsche Polynome', J. Reine. Angew. Appl. Math. 150 (1920), 79-106.

[6] P. Fatou, 'Sur les équations fonctionelles', Bull. Soc. Math. France 47 (1919), 161-271; 48 (1920), 33-94, 208-314.

[7] B. Fischer, 'Chebyshev polynomials for disjoint compact sets', Constr. Approx. 8 (1992), 309-329.

[8] G. Julia, 'Mémoire sur l'itération des fonctions rationelles', J. Math. Pures Appl. Ser. 8(1) (1918), 47-245.

[9] J. Milnor, Dynamics in One Complex Variable. Introductory Lectures (Vieweg, Braunschweig, 1999). 
[9] Chebyshev polynomials on Julia sets and equipotential curves for the family $P(z)=z^{d}-c$

[10] P. Montel, Leçons sur les familles normales de fonctions analytiques et leurs applications (Gauthier-Villars, Paris, 1927).

[11] M. Stawiska, 'Chebyshev polynomials on equipotential curves of a quadratic Julia set', Univ. Iagel. Acta Math. 33 (1996), 191-198.

YINGQING XIAO, School of Mathematical Sciences, Fudan University, Shanghai 200433, People's Republic of China

and

Current address: College of Mathematics and Econometrics, Hunan University, Changsha 410082, People's Republic of China

e-mail: xiaoyingqing@yahoo.com.cn

WEIYUAN QIU, School of Mathematical Sciences, Fudan University, Shanghai 200433, People's Republic of China

e-mail: wyqiu@fudan.edu.cn 